\title{
Charm Physics with BES-III at BEPC-II
}

\author{
Haibo $\mathrm{Li}^{\mathrm{a} *}$ \\ ${ }^{a}$ Institute of High Energy Physics, P.O.Box 918, Beijing 100049, China
}

\begin{abstract}
We report on the charm physics potential at BES-III at BEPC-II which will make significant contribution to quark flavor physics this decade. The charm physics program will include studies of leptonic, semileptonic and hadronic charm decays, and tests for physics beyond the Standard Model. Event samples of the order of 30 million $D \bar{D}$ pairs, 2 million $D_{S}^{+} D_{S}^{-}$pairs at threshold and $10 \times 10^{9} \mathrm{~J} / \psi$ decays will be produced with one year design luminosity. High precision charm data will enable us to validate forthcoming Lattice QCD calculations at the few percent level. These can then be used to make precise measurements of CKM elements, $V_{c d}, V_{c s}, V_{u b}, V_{c b}$ and $V_{t s}$, which are useful to improve the accuracy of test of the CKM unitarity.
\end{abstract}

\section{Introduction}

BES-III at BEPC-II under construction in Beijing will focus on charm and QCD physics in the range $\sqrt{s}=2.0-4.2 \mathrm{GeV}$. The physics program at BES-III includes a variety of measurements that will improve the understanding of the Standard Model (SM) processes as well as provide the opportunity to probe for physics that lies beyond the SM. However, the possibility of new physics may be diluted by the large hadronic uncertainty in $D$ decays. The study of weak interactions in the charm sector, and the extraction of quark mixing matrix parameters remain limited by our capacity to cope with nonperturbative QCD. Thus, it will be of great benefit if we can understand the effects of strong coupling in QCD. High precision predictions of QCD will then remove road blocks for many weak and flavor physics measurements.

Lattice QCD (LQCD) [1 had matured over the last decade. Recent advances in LQCD have produced a wide variety of calculations of nonperturbative quantities with accuracies in the 10$20 \%$ level for $D$ decay constant and form factors. We finally can expect the first LQCD results with $1-3 \%$ errors. BES-III will provide crucial data to validate them and help guide the development of QCD calculation techniques for a full understanding of non-perturbative QCD effects. This

\footnotetext{
*Email: lihb@ihep.ac.cn
}

will improve the measurements of charm meson decay constants, $V_{c s}$ and $V_{c d}$ to $1 \%$ level.

Table 1

$\tau$-Charm productions at BEPC-II in one year's running $\left(10^{7} \mathrm{~s}\right)$.

\begin{tabular}{lll}
\hline Data Sample & $\begin{array}{l}\text { Central-of-Mass } \\
(\mathrm{MeV})\end{array}$ & $\begin{array}{l}\text { \#Events } \\
\text { per year }\end{array}$ \\
\hline$J / \psi$ & 3097 & $10 \times 10^{9}$ \\
$\tau^{+} \tau^{-}$ & 3670 & $12 \times 10^{6}$ \\
$\psi(2 S)$ & 3686 & $3.0 \times 10^{9}$ \\
$D^{0} \bar{D}^{0}$ & 3770 & $18 \times 10^{6}$ \\
$D^{+} D^{-}$ & 3770 & $14 \times 10^{6}$ \\
$D_{S}^{+} D_{S}^{-}$ & 4030 & $1.0 \times 10^{6}$ \\
$D_{S}^{+} D_{S}^{-}$ & 4170 & $2.0 \times 10^{6}$ \\
\hline
\end{tabular}

Beginning in mid-2007, the BEPC-II accelerator will be operated at center-of-mass $(\mathrm{CM})$ energies corresponding to $\sqrt{s}=2.0-4.2 \mathrm{GeV}$. The designed luminosity over this energy region will range from $1 \times 10^{33} \mathrm{~cm}^{-2} \mathrm{~s}^{-1}$ down to about $0.6 \times 10^{33} \mathrm{~cm}^{-2} \mathrm{~s}^{-1}\left[2\right.$, yielding around $5 \mathrm{fb}^{-1}$ each at $\psi(3770)$ and at $\sqrt{s}=4170 \mathrm{MeV}$ [3] above $D_{S}^{+} D_{S}^{-}$threshold and $3 \mathrm{fb}^{-1}$ at $J / \psi$ peak in one year's running with full luminosity [2]. These integrated luminosities correspond to samples of 2.0 million $D_{S}^{+} D_{S}^{-}, 30$ million $D \bar{D}$ pairs and $10 \times 10^{9}$ $J / \psi$ decays. Table $\prod$ summarizes the data set per year at BES-III. In this report, all preliminary 
sensitivity studies are based on $20 \mathrm{fb}^{-1}$ luminosity at $\psi(3770)$ peak for $D$ physics, the same luminosity also for $D_{S}$ physics at $\sqrt{s}=4170 \mathrm{MeV}$.

\section{Unique Features of the Charm Physics at BES-III}

Many of the measurements related to charm decays have been done by other experiments such as BES-II and CLEO-c, and many are also accessible to the B-factory experiments. What are BES-III's advantages to running at the open charm threshold?

Firstly, compared to the old BES experiment, BES-III is a state-of-the-art detector with modern technologies 2. BES-I/BES-II was modeled on MARK-III detector. It has gone through several generations of development since then. In every resolution and performance parameter, for example, hit resolution, momentum and energy resolutions, mass resolution, particle identification (PID) capability, solid angle coverage, BES-III is superior to previous versions of BES detectors by substantial margins. The new detector consists of a He-based small cell drift chamber, Time-OfFlight (TOF) counters for PID, a CsI(Tl) crystal calorimeter, a solenoid super-conducting magnet with a field of 1 Tesla and the magnet yoke interleaved with RPC counters as the muon chamber. The construction is expected to be completed in the middle of 2007. Photon energy resolution is $\Delta E / E=2.5 \%$ at $E_{\gamma}=1.0 \mathrm{GeV}$. The momentum resolution is $\sigma_{p} / p=0.5 \%$ at $p=1.0 \mathrm{GeV} / c$, and the $d E / d x$ resolution for hadron tracks is about $6 \%$. The time resolution of TOF is about $100 \mathrm{ps}$, combining energy loss $(d E / d x)$ measurement in the draft chamber, give $10 \sigma \mathrm{K} / \pi$ resolution across the typical kinematic range. A $25 \%$ increase of solid angle coverage relative to BES improve efficiency for double-tag measurements greatly. The gains go as $1.25^{N}$, where $N$ is the total number of tracks and photons of the event 4 . This indicates a typical effective luminosity gain of 8 in such analyses. For partial wave analysis, the increase in solid angle coverage means angular distributions will be measured across the almost full angular range without large variations in acceptance. The $J^{P C}$ and partial waves can be measured reliably and precisely.

Secondly, BES-III will not be able to compete both BaBar and Belle in statistics on charm physics, especially on the rare and forbidden decays of charm mesons. However, data taken at charm threshold still have powerful advantages over the data at $\Upsilon(4 S)$, which we list here [4]: 1) Charm events produced at threshold are extremely clean; 2) The measurements of absolute branching fraction can be made by using double tag events; 3) Signal/Background is optimum at threshold; 4) Neutrino reconstruction is clean; 5) Quantum coherence allow simple [5] and complex [6] methods to measure $D^{0} \bar{D}^{0}$ mixing parameters and check for direct $C P$ violation.

\section{Charm Decays and Production Cross Sections at BEPC-II}

The main targets of the charm physics program at BES-III are absolute branching fraction measurements of leptonic, semileptonic and hadronic decays. The first measures decay constants and the second measures form factors and, in combination with theory, allows the determination of $V_{c s}$ and $V_{c d}$. The third of those provides an absolute scale for all charm and hence beauty decays.

At $D \bar{D}$ or $D_{S}^{+} D_{S}^{-}$threshold, no additional hadrons accompanying the $D \bar{D}$ or $D_{S}^{+} D_{S}^{-}$pairs are produced. Reconstruction of one $D$ or $\bar{D}$ meson (called single tag or ST) tags the event as either $D^{0} \bar{D}^{0}$ or $D^{+} D^{-}\left(D_{S}^{+} D_{S}^{-}\right)$. For a given decay mode $i$, we measure independently the $D$ and $\bar{D}$ ST yields, denoted by $N_{i}$ and $\bar{N}_{i}$. We determine the corresponding efficiencies from Monte Carlo simulations (MC), denoted by $\epsilon_{i}$ and $\bar{\epsilon}_{i}$. Thus, $N_{i}=\epsilon_{i} \mathcal{B}_{i} N_{D \bar{D}}$ and $\bar{N}_{i}=\bar{\epsilon}_{i} \mathcal{B}_{i} N_{D \bar{D}}$, where $\mathcal{B}_{i}$ is the branching fraction for mode $i$, assuming no $C P$ violation, and $N_{D \bar{D}}$ is the total number of produced $D \bar{D}$ pairs at BES-III. Double tag (DT) events are the subset of ST events where both the $D$ and $\bar{D}$ are reconstructed. The DT yield for $D$ mode $i$ and $\bar{D}$ mode $j$, denoted by $N_{i j}$, is given by $N_{i j}=\epsilon_{i j} \mathcal{B}_{i} \mathcal{B}_{j} N_{D \bar{D}}$, where $\epsilon_{i j}$ is the DT efficiency. As with ST yields, the charge conjugate DT yields and efficiencies, $N_{j i}$ and $\epsilon_{j i}$, are determined separately. Charge conjugate particles are implied, unless referring to ST and DT 
yields. The absolute branching fraction $\mathcal{B}_{i}$ can be obtained from $\mathcal{B}_{i}=\frac{N_{i j}}{\bar{N}_{j}} \frac{\bar{\epsilon}_{j}}{\epsilon_{i j}}$. With the same method, we have the total number of $D \bar{D}$ pairs $N_{D \bar{D}}=\frac{N_{i} \bar{N}_{j}}{N_{i j}} \frac{\epsilon_{i j}}{\left(\epsilon_{i} \bar{\epsilon}_{j}\right)}$, which can be used to obtain the absolute cross-section of $D \bar{D}$ production.

\subsection{Leptonic Charm Decays}

From the leptonic decays of $D^{ \pm}$and $D_{S}^{ \pm}$ mesons, the decay constants $f_{D}$ and $f_{D_{S}}$ can be determined to a precision of about $1 \%$. The decay constants measure the non-perturbative wave function of the meson at zero inter-quark separation and appear in all processes where constituent quarks must approach each other at distances small compared to the meson size 4].

Table 2

Expected errors on the branching fractions for leptonic decays and decays constants at BES-III with $20 \mathrm{fb}^{-1}$ at $\psi(3770)$ peak.

\begin{tabular}{lll}
\hline Decay Modes & $\begin{array}{l}\text { Error }(\%) \\
\text { on } \mathcal{B}\end{array}$ & $\begin{array}{l}\text { Error }(\%) \\
\text { on } f_{D(s)}\end{array}$ \\
\hline$D^{+} \rightarrow \mu^{+} \nu\left(f_{D}\right)$ & 2.0 & 1.5 \\
$D_{S}^{+} \rightarrow \mu^{+} \nu\left(f_{D_{S}}\right)$ & 2.0 & 1.1 \\
$D_{S}^{+} \rightarrow \tau^{+} \nu\left(f_{D_{S}}\right)$ & 1.5 & 0.9 \\
\hline
\end{tabular}

Measurements of leptonic decays at BES-III will benefit from the fully tagged $D^{+}$and $D_{S}^{+}$decays available at the $\psi(3770)$ and at $\sqrt{s} \sim 4170$ $\mathrm{MeV}$ [3]. The leptonic decay of $D^{+}\left(D_{S}^{+}\right) \rightarrow \mu^{+} \nu$ is detected in tagged events by observing a single charged track of the correct sign, missing energy, and a complete accounting of the residual energy in the calorimeter. The pure $D \bar{D}$ pair in the initial state and cleanliness of the full tag reconstruction make this measurement essentially background-free. The leptonic decay rates for $D^{+}$and $D_{S}^{+}$can be measured with a precision of $1-2 \%$ level. This will allow the validation of theoretical calculations of the decay constants at the 1\% level. Table 2 summarizes the expected precision in the decay constant measurements. It should be noted that the $D^{+} \rightarrow \tau^{+} \nu$ decay is re- ported by CLEO-c with upper limit of $2.1 \times 10^{-3}$ at $90 \% \mathrm{CL}$ [7. At BES-III, the sensitivity will be $10^{-5}-10^{-6}$ level.

\subsection{Semileptonic Charm Decays}

Semileptonic widths for $D \rightarrow X_{s(d)} l^{+} \nu$ directly probe the elements of the CKM matrix. When $J^{P}\left(X_{s, d}\right)=0^{-}$, the differential width is given by:

$\frac{d \Gamma\left(D \rightarrow X_{s(d)} l^{+} \nu\right)}{d q^{2}}=\frac{G_{F}^{2}}{24 \pi^{3}} p^{3}\left|V_{c s(d)}\right|^{2} p^{3}\left|F\left(q^{2}\right)\right|^{2}$,

where $q^{2}$ is the momentum transfer squared between the $D$ meson and the final state hadron in the $D$ rest frame, and $F\left(q^{2}\right)$ is the hadronic form factor at $c \rightarrow W s(d)$ vertex. The form factor can be predicted from a number of different theoretical approaches [8] including LQCD [1]. In addition to its own intrinsic interest, the analogous form factor is needed for extracting $b$-quark matrix elements such as $V_{u b}$ in $B$ semileptonic decays, so it is important to demonstrate the reliability of any one calculation.

Absolute branching ratios in critically interesting modes, such as, $D \rightarrow \pi l \nu, D \rightarrow K l \nu$, $D \rightarrow \eta\left(\eta^{\prime}\right) l \nu, D \rightarrow \rho l \nu, D \rightarrow K^{*} l \nu, D_{S} \rightarrow \phi l \nu$ and $D_{S} \rightarrow K^{*} l \nu$, will be measured to be less than $1 \%$, and the form factor slopes to $1.5 \%$. The measurement in each case is based on the use of tagged events where the cleanliness of the environment provides nearly background-free signal samples. This will lead to the determination of the CKM matrix elements $V_{c s}$ and $V_{c d}$ with a precision of $1 \%$ level assuming knowledge of the relevant form factors with $1.5 \%$ uncertainties from LQCD. Form factors in all modes can be measured across the full range of $q^{2}$ with excellent resolution. Measurements of the vector and axialvector form factors $V\left(q^{2}\right), A_{1}\left(q^{2}\right)$ and $A_{2}\left(q^{2}\right)$ will also be possible at the $\sim 2 \%$ level. Table 3 summarizes the most recent results and the expected fractional errors on the branching ratios at BESIII.

With high statistics of $D$ sample at BES-III, many unobserved semileptonic $D$ decays will be accessible, such as $D+\rightarrow f_{0} l \nu, D+\rightarrow \eta\left(\eta^{\prime}\right) l \nu$, $D^{+} \rightarrow \bar{K}^{* *} l \nu$ and $D^{+} \rightarrow \phi l \nu$, where $\bar{K}^{* *}$ is the exited koan mesons including $\bar{K}_{1}(1270)$, $\bar{K}^{*}(1410)$ and $\bar{K}^{*}(1430)$. These measurements 
Table 3

Uncertainties on the branching fractions for $D$ and $D_{S}$ semileptonic decay modes and precision of form factor parameters at BES-III (assuming $20 \mathrm{fb}^{-1}$ data at $\psi(3770)$ peak). The precision of parameters is mainly limited by the uncertainties on $V_{c d}$ and $V_{c s}$.

\begin{tabular}{lllll}
\hline Decay Modes & $\begin{array}{l}\text { Error (\%) } \\
\text { on } \mathcal{B} \text { PDG2004 }\end{array}$ & $\begin{array}{l}\text { Expected Error (\%) } \\
\text { on } \mathcal{B} \text { at BES-III }\end{array}$ & $\begin{array}{l}\text { Form Factor } \\
\text { Type }\end{array}$ & $\begin{array}{l}\text { Expected Error (\%) } \\
\text { on Form factor at BES-III }\end{array}$ \\
\hline$D^{0} \rightarrow K l \nu$ & 4.6 & 0.2 & PS $\rightarrow$ PS & 1.0 \\
$D^{0} \rightarrow \pi l \nu$ & 9.6 & 0.4 & PS $\rightarrow$ PS & 1.0 \\
$D^{+} \rightarrow \pi l \nu$ & 50.0 & 0.8 & PS $\rightarrow$ PS & 1.0 \\
$D^{+} \rightarrow \bar{K}^{*} l \nu$ & 10.0 & 0.3 & PS $\rightarrow$ V & 2.0 \\
$D_{S}^{+} \rightarrow \phi l \nu$ & 25.0 & 1.2 & PS $\rightarrow$ V & 1.0 \\
\hline
\end{tabular}

will provide another lab to study the $K \pi$ and $\pi \pi$ S-wave [9].

The ratio of decay widths $\Gamma\left(D_{S} \rightarrow\right.$ $\left.\eta^{\prime} e \nu\right) / \Gamma\left(D_{S} \rightarrow \eta e \nu\right)$, using $\left|V_{c s}\right|=0.975$ [10,

$\frac{\Gamma\left(D_{S} \rightarrow \eta^{\prime} e \nu\right)}{\Gamma\left(D_{S} \rightarrow \eta e \nu\right)} \sim 0.28 \times|\cot \phi|^{2}$,

depends on the content of the $\eta$ and $\eta^{\prime}$ mesons, where $\phi$ is the mixing angle in the $\eta-\eta^{\prime}$ flavor mixing scheme 11. We note that the decays $D_{S} \rightarrow \eta\left(\eta^{\prime}\right) l \nu$ involve the strange content of $\eta\left(\eta^{\prime}\right)$, and $D^{+} \rightarrow \eta\left(\eta^{\prime}\right) l \nu$ involve the non-strange content. Therefore, $D^{+} \rightarrow \eta\left(\eta^{\prime}\right) l \nu$ and $D_{S} \rightarrow \eta\left(\eta^{\prime}\right) l \nu$ could provide combined testing of a $\eta-\eta^{\prime}$ mixing scheme [12]. The uncertainty on mixing angle $\phi$ is about $13 \%$ with current $D_{S}^{+}$data [1], at BES-III, the error will be $2 \%$ level.

\subsection{Absolute Hadronic Branching Frac- tion and $e^{+} e^{-} \rightarrow D \bar{D}, D \bar{D}^{*}$, and $D^{*} \bar{D}^{*}$ Cross Sections}

Absolute branching fraction measurements are important since, for a lot of analyses at higher energies as well as in the $B$-system, an inaccurate knowledge of $D, D_{S}$ decays can result in large systematic errors. Using double-tagged events at threshold leaves only major systematic error contributions from efficiency uncertainties in the tracks and showers.

The rate for the critical normalizing modes $D \rightarrow K \pi, D^{+} \rightarrow K \pi \pi$, and $D_{S} \rightarrow \phi \pi$ will be established to a precision of order less than 1.0\%. At BES-III, the statistic is high enough that we can measure Cabibbo-suppressed decays of $D$ mesons, especially the $D \rightarrow 4 \pi, 5 \pi$ or even
Table 4

Unobserved 3-body $D$ decays, $D^{0} \rightarrow P^{0} P^{0} X^{0}$, where $P^{0}$ is pseudoscalar and $X^{0}$ is any kind of particle allowed in the final states. $f_{0}$ and $a_{0}$ are the scalar $f_{0}(980)$ and $a_{0}(980)$, respectively. N/A represents not available.

\begin{tabular}{c|c|c|c|c}
\hline & \multicolumn{4}{|c}{$P^{0}$} \\
\hline$X^{0}$ & $\pi^{0}$ & $\eta$ & $K_{S}$ & $K_{L}$ \\
\hline$\pi^{0}$ & $\pi^{0} \pi^{0} \pi^{0}$ & $\eta \eta \pi^{0}$ & $K_{S} K_{S} \pi^{0}$ & $K_{L} K_{L} \pi^{0}$ \\
\hline$\eta$ & $\pi^{0} \pi^{0} \eta$ & $\eta \eta \eta$ & $K_{S} K_{S} \eta$ & $K_{L} K_{L} \eta$ \\
\hline$\eta^{\prime}$ & $\pi^{0} \pi^{0} \eta^{\prime}$ & $\mathrm{N} / \mathrm{A}$ & $\mathrm{N} / \mathrm{A}$ & N/A \\
\hline$K_{S}$ & $\pi^{0} \pi^{0} K_{S}$ & $\eta \eta K_{S}$ & $K_{S} K_{S} K_{S}$ & $K_{L} K_{L} K_{S}$ \\
\hline$K_{L}$ & $\pi^{0} \pi^{0} K_{L}$ & $\eta \eta K_{L}$ & $K_{S} K_{S} K_{L}$ & $K_{L} K_{L} K_{L}$ \\
\hline$a_{0}$ & $\pi^{0} \pi^{0} a_{0}$ & N/A & N/A & N/A \\
\hline$f_{0}$ & $\pi^{0} \pi^{0} f_{0}$ & N/A & N/A & N/A \\
\hline
\end{tabular}

more pions final states [13. The sensitivities of the measurements of these branching fractions will be $10^{-5}-10^{-6}$ level at BES-III. Many unobserved 3-body $D$ decays can also be observed at BES-III as listed in Table 4 One should note that the decays $D\left(D_{S}\right) \rightarrow V_{1} V_{2}$ are very important to probe the final state interaction by measuring the polarization fractions, $f_{L}, f_{T}\left(f_{\perp}\right.$ and $\left.f_{\|}\right)$. At BES-III, these measurements will become available.

As discussed at the beginning of Section 3 we can obtain the $e^{+} e^{-} \rightarrow D \bar{D}$ cross sections by scaling $N_{D^{0} \bar{D}^{0}}$ and $N_{D^{+} D^{-}}$by the luminosity at each energy point. At $E_{c m}=3773 \mathrm{MeV}$, CLEO-c collaboration found peak cross sections of $\sigma\left(e^{+} e^{-} \rightarrow D^{0} \bar{D}^{0}\right)=\left(3.60 \pm 0.07_{-0.05}^{+0.07}\right) \mathrm{nb}$, $\sigma\left(e^{+} e^{-} \rightarrow D^{+} D^{-}\right)=\left(2.79 \pm 0.07_{-0.04}^{+0.10}\right) \mathrm{nb}$, 
$\sigma\left(e^{+} e^{-} \rightarrow D \bar{D}\right)=\left(6.39 \pm 0.10_{-0.08}^{+0.17}\right) \mathrm{nb}$, and the ratio of charged $D$ pairs and neutral $D$ pairs production is about $0.776 \pm 0.024_{-0.006}^{+0.014}$ [14, where the uncertainties are statistical and systematic, respectively. The ratio is significantly deviated from one in $D \bar{D}$ pair production near threshold. It is mainly due to the substantial mass difference between the charged and neutral $D$ mesons, which will produce a factor of $\frac{p_{+-}^{3}}{p_{00}^{3}}=0.69$, where $p_{+-}$and $p_{00}$ are the momentum of charged and neutral $D$ mesons in $\psi(3770)$ rest frame. Recently, Voloshin pointed out [15] that this ratio should exhibit a prominent variation across the $\psi(3770)$ resonance due to the interference of the resonance scattering phase with the Coulomb interaction between the charged $D$ mesons. The energy dependent ratio $R$ is expressed by [15] :

$R\left(E_{c m}\right)=\frac{\sigma\left(e^{+} e^{-} \rightarrow D^{+} D^{-}\right)}{\sigma\left(e^{+} e^{-} \rightarrow D^{0} \bar{D}^{0}\right)}=F_{c} \frac{p_{+-}^{3}}{p_{00}^{3}}$,

where $F_{c}$ is the correction factor of the Coulomb interaction, it is a function of relative phase of electromagnetic and strong interactions as described in ref. [15]. The expected variation of the ratio in the vicinity of $\psi(3770)$ peak is about a few percent level, which may be sufficient for a study in the upcoming BES-III experiment.

It is quite well known 16 that the cross sections of $e^{+} e^{-} \rightarrow D^{*} \bar{D}^{*}, D^{*} \bar{D}, D_{S}^{*+} D_{S}^{*-}$ and $D_{S}^{*+} D_{S}^{-}$in the region just above the threshold of open charm production around $4.0 \mathrm{GeV}$ display an intricate behavior which is yet to be studied in detail. This behavior is caused by the successive onset of specific channels with the $D$ mesons by the strong dynamics in each of these channels and by the coupling between them 17. Thus, a detailed experimental study of this region at BES-III will provide rich information about the strong dynamics of systems with heavy and light quarks.

\section{4. $\psi(3770)$ non- $D \bar{D}$ Decays}

It is well known that the $\psi(3770)$ decays most copiously into the OZI-allowed $D \bar{D}$ pair owing to the closeness of the mass threshold. Hadronic or radiative transitions to lower-lying $c \bar{c}$ states, decay to lepton pairs, or decay to light hadrons are all available and predicted 181920, but their branching fractions are highly suppressed. BESII reported the first signal of non- $D \bar{D}$ decays of $\psi(3770)$, at $\sim 3 \sigma$ significance, with $\mathcal{B}(\psi(3770) \rightarrow$ $\left.\pi^{+} \pi^{-} J / \psi\right)=(0.34 \pm 0.14 \pm 0.09) \%$ [21]. CLEOc has also searched for the non- $D \bar{D}$ decays of $\psi(3770)$, including $\pi \pi J / \psi, \gamma \chi_{c 1}$ and light hadron final states 22. The CLEO-c collaboration measured cross section for $e^{+} e^{-} \rightarrow \psi(3770) \rightarrow$ hadrons at $E_{c m}=3773 \mathrm{MeV}$ to be $(6.38 \pm$ $\left.0.08_{-0.30}^{+0.41}\right) \mathrm{nb}[23$. The difference between this and the $e^{+} e^{-} \rightarrow \psi(3770) \rightarrow D \bar{D}$ cross section at the same energy is found to be $\left(-0.01 \pm 0.08_{-0.30}^{+0.41}\right)$ nb, which indicates the non- $D \bar{D}$ decays would be smaller than $0.53 \mathrm{nb}$ at $90 \% \mathrm{CL}$, or corresponds to an upper limit of $8.4 \%$ of the branching fraction at $90 \%$ CL. BESII also reported an upper limit of $30 \%$ of the branching fraction at $90 \%$ CL 24 .

In order to understand the decay dynamics of non- $D \bar{D}$ decays and the line-shape of $\psi(3770)$ resonance, it is very important to measure the cross sections of inclusive hadron productions by using scan method in the vicinity of $\psi(3770)$ peak. At BES-III, the sensitivity to non- $D \bar{D}$ measurement is estimated to be less than $1 \%$ level by using scan method with one year integrated luminosity at different energy points near $\psi(3770)$ peak. A detailed MC simulation is in process.

The exclusive charmless $\psi(3770)$ decay modes should be searched at BES-III. The sensitivity to exclusive charmless $\psi(3770)$ decay modes at BESIII will be around $10^{-6}-10^{-7}$ with $20 \mathrm{fb}^{-1}$ data. One has to consider the interference between resonances and continuum, and also the interference between different resonances near $\psi(3770)$ [25].

\section{4. $D^{0}-\bar{D}^{0}$ Mixing, $C P$ Violation and Physics Beyond the Standard Model}

With the design luminosity of $10^{33} \mathrm{~cm}^{-2} \mathrm{~s}^{-1}$, BES-III will have the opportunity to probe for the possible new physics which may enter uptype-quark decays. It includes searches for charm mixing, $C P$ violation and rare charm decays. The BES-III charm physics program also includes a variety of measurements that will improve the determination of $\phi_{3} / \gamma$ from $B$-factory experiments. The total number of charm mesons accumulated 
at BES-III will be much smaller than that at $B$ factories which are about $500 \mathrm{fb}^{-1}$ for each of them. However, the quantum correlations in the $\psi(3770) \rightarrow D \bar{D}$ system will provide a unique laboratory in which to study charm $[$.

\section{1. $D^{0}-\bar{D}^{0}$ Mixing and $C P$ Violation}

$D^{0}-\bar{D}^{0}$ mixing within the SM are highly suppressed due to GIM mechanism, thus, at BES-III, searches for neutral charm mixing and $C P$ violation in charm decays may be essential in deciding if some intriguing signals are actually due to new physics.

The time evolution of $D^{0}-\bar{D}^{0}$ system, assuming no $C P$ violation in mixing, is governed by four parameters: $x=\Delta m / \Gamma$ and $y=\Delta \Gamma / 2 \Gamma$ which are the mass and width differences of $D$ meson mass eigenstates and characterize the mixing matrix, $\delta$ the relative strong phase between Cabibbo favor (CF) and doubly-Cabibbo suppressed (DCF) amplitudes and $R_{D}$ the DCF decay rate relative to the $\mathrm{CF}$ decay rate. The mixing rate $R_{M}$ is defined as $\frac{1}{2}\left(x^{2}+y^{2}\right)$ [26]. Standard Model based predictions for $x$ and $y$, as well as a variety of non-Standard Model expectations, span several orders of magnitude 26] which is $x$ $\sim y \sim 10^{-3}$. Presently, experimental information about charm mixing parameters $x$ and $y$ comes from the time-dependent analyses. The current experimental upper limits on $x$ and $y$ are on the order of a few times $10^{-2}$.

At BES-III, time-dependent analyses are not available. However, one can use the fact that $D^{0} \bar{D}^{0}$ pairs in $\psi(3770) \rightarrow D \bar{D}$ decays have the useful property that the two mesons are in the $C P$-correlated states [6], namely, one $D$ state decayed into the final state with definite $C P$ properties immediately identifies or tags $C P$ properties of the state on the other side. It provides timeintegrated sensitivity to $R_{M}$ at $10^{-4}$ level by considering the decay $\psi(3770) \rightarrow\left(K^{-} \pi^{+}\right)\left(K^{-} \pi^{+}\right)$ hadronic final state only at BES-III. We have not estimated the double semileptonic channel yet, $\psi(3770) \rightarrow\left(l^{ \pm}(K X)\right)\left(l^{ \pm}(K X)\right)$. Sensitivity to $\cos \delta$ is 0.03 for $D^{0} \rightarrow K \pi$ mode. Recently, a simultaneous determinations of the mixing, relative strong phase and $R_{D}$ have been proposed by using various single tag, double tag and $C P$ tag rates at CLEO-c 6]. The sensitivities on $x$ and $y$ are improved greatly, but we have not estimated this method at BES-III yet.

For the direct $C P$ violation, the SM predictions are as large as $0.1 \%$ for $D^{0}$ decays, and $1 \%$ level for $D^{+}$and $D_{S}$ decays 27 . At BES-III, one can also look at the $C P$ violation by exploiting the quantum coherence at the $\psi(3770)$. Consider the case where both the $D^{0}$ and the $\bar{D}^{0}$ decay into $C P$ eigenstates, then the decays $\psi(3770) \rightarrow f_{+}^{i} f_{+}^{i}$ or $f_{-}^{i} f_{-}^{i}$ are forbidden, where $f_{+}\left(f_{-}\right)$denotes a $C P+$ eigenstate $(C P-$ eigenstate). This is because $C P\left(f_{ \pm}^{i} f_{ \pm}^{i}\right)=C P\left(f_{ \pm}^{i}\right) C P\left(f_{ \pm}^{i}\right)(-1)^{l}=-1$, while, for the $l=1 \psi(3770)$ state, $C P(\psi(3770))=$ +1 . Thus observation of a final state such as $\left(K^{+} K^{-}\right)\left(\pi^{+} \pi^{-}\right)$constitutes evidence of $C P$ violation. For $\left(K^{+} K^{-}\right)\left(\pi^{+} \pi^{-}\right)$mode, the sensitivity at BES-III is about $1 \%$ level. Moreover, all pairs of $C P$ eigenstates, where both eigenstates are even or both are odd, can be summed over for $C P$ violation measurements at BES-III.

\subsection{Dalitz Plot Analyses}

Recent studies of multi-body decays of $D$ mesons provide a direct probe of the final state interactions by looking at the interference between intermediate state resonances on the Dalitz Plot (DP). When $D$ mesons decays into three or more daughters, intermediate resonances dominate the decay rates. These resonances will cause a nonuniform distribution of events in phase space on the DP. Since all events on the DP have the same final states, different resonances at the same location on DP will interfere. This provides the opportunity to measure both the amplitudes and phases of the intermediate decay channels, which in turn allows to deduce their relative branching fractions. These phase differences can even allow details about very broad resonances to be extracted by observing their interferences with other intermediate states.

The most important thing is that recent studies of multi-body decays of $D$ mesons probe a variety of physics including light spectroscopy $(\pi \pi$, $K \pi$ and $K K \mathrm{~S}$-wave states), searches for $C P$ violation and $D^{0}-\bar{D}^{0}$ mixing. Currently, the decay $D^{0} \rightarrow K_{S} \pi^{+} \pi^{-}$plays very important role in 
the determination of $\phi_{3} / \gamma$. Recently BaBar and Belle 28 have reported $\gamma=\left(70 \pm 31_{-10-11}^{+12+14}\right)^{o}$ and $\phi_{3}=\left(77_{-19}^{+17} \pm 13 \pm 11\right)^{\circ}$, respectively, where the third error is the systematic error due to modeling of DP. The precision of these measurements will eventually be limited by the understanding of the $D^{0} \rightarrow K_{S} \pi^{+} \pi^{-}$decays. Although K-matrix description of the $\pi \pi \mathrm{S}$-wave may yield improved models of the DP and the error on $\phi_{3} / \gamma$ may be decreased from $\pm 10^{\circ}$ to a few degrees, it is still a model-dependent way to extract the angle. At BES-III, by using the coherence of $D^{0} \bar{D}^{0}$ pairs at $\psi(3770)$ peak, one can study the CP-tagged and flavor-tagged DP by doing binned analysis [29]. This method is a model-independent. According to the estimation in reference 29], the proposed super- $B$ factory [30] with its design integrated luminosity of $50 \mathrm{ab}^{-1}$, would allow a measurement of $\phi_{3} / \gamma$ with accuracy below $2^{\circ}$. To keep the uncertainty due to $D$ DP decays below that level, around $10^{-4} C P$-tagged $D$ decays are needed, corresponding to $\sim 10 \mathrm{fb}^{-1}$ data which can be obtained at BEPC-II with two years' luminosity.

Table 5

Current and projected 90\%-CL upper limits on rare $D^{+}$decay modes at BES-III with $20 \mathrm{fb}^{-1}$ data at $\psi(3770)$ peak. We assume the selection efficiencies for all modes are $35 \%$.

\begin{tabular}{llll}
\hline Mode & $\begin{array}{l}\text { Reference } \\
\text { Experiment }\end{array}$ & $\begin{array}{l}\text { Best Upper } \\
\text { limits }\left(10^{-6}\right)\end{array}$ & $\begin{array}{l}\text { BES-III } \\
\left(\times 10^{-6}\right)\end{array}$ \\
\hline$\pi^{+} e^{+} e^{-}$ & CLEO-c [31] & 7.4 & 0.03 \\
$\pi^{+} \mu^{+} \mu^{-}$ & FOCUS [32] & 8.8 & 0.03 \\
$\pi^{+} \mu^{+} e^{-}$ & E791 [33] & 34 & 0.03 \\
$\pi^{-} e^{+} e^{+}$ & CLEO-c [31] & 3.6 & 0.03 \\
$\pi^{-} \mu^{+} \mu^{+}$ & FOCUS [32] & 4.8 & 0.03 \\
$\pi^{-} \mu^{+} e^{+}$ & E791 [33] & 50 & 0.03 \\
$K^{+} e^{+} e^{-}$ & CLEO-c [31] & 6.2 & 0.03 \\
$K^{+} \mu^{+} \mu^{-}$ & FOCUS [32] & 9.2 & 0.03 \\
$K^{+} \mu^{+} e^{-}$ & E791 [33] & 68 & 0.03 \\
$K^{-} e^{+} e^{+}$ & CLEO-c [31] & 4.5 & 0.03 \\
$K^{-} \mu^{+} \mu^{+}$ & FOCUS [32] & 13 & 0.03 \\
$K^{-} \mu^{+} e^{+}$ & E687 [34] & 130 & 0.03 \\
\hline
\end{tabular}

Table 6

Current and projected 90\%-CL upper limits on rare $D^{0}$ decay modes at BES-III with $20 \mathrm{fb}^{-1}$ data at $\psi(3770)$ peak.

\begin{tabular}{llll}
\hline Mode & $\begin{array}{l}\text { Reference } \\
\text { Experiment }\end{array}$ & $\begin{array}{l}\text { Best Upper } \\
\text { limits }\left(10^{-6}\right)\end{array}$ & $\begin{array}{l}\text { BES-III } \\
\left(\times 10^{-6}\right)\end{array}$ \\
\hline$\gamma \gamma$ & CLEO [37] & 28 & 0.05 \\
$\mu^{+} \mu^{-}$ & D0 [39] & 2.4 & 0.03 \\
$\mu^{+} e^{-}$ & E791 [33] & 8.1 & 0.03 \\
$e^{+} e^{-}$ & E791 [33] & 6.2 & 0.03 \\
$\pi^{0} \mu^{+} \mu^{-}$ & E653 [40] & 180 & 0.05 \\
$\pi^{0} \mu^{+} e^{+}$ & CLEO [38] & 86 & 0.05 \\
$\pi^{0} e^{+} e^{-}$ & CLEO [38] & 45 & 0.05 \\
$K_{S} \mu^{+} \mu^{-}$ & E653 [40] & 260 & 0.1 \\
$K_{S} \mu^{+} e^{-}$ & CLEO [38] & 100 & 0.1 \\
$K_{S} e^{+} e^{-}$ & CLEO [38] & 110 & 0.1 \\
$\eta \mu^{+} \mu^{-}$ & CLEO [38] & 530 & 0.1 \\
$\eta \mu^{+} e^{-}$ & CLEO [38] & 100 & 0.1 \\
$\eta e^{+} e^{-}$ & CLEO [38] & 110 & 0.1 \\
\hline
\end{tabular}

\subsection{Rare Charm Decays}

Searches for rare-decay processes have played an important role in the development of the SM. Short-distance flavor-changing neutral current (FCNC) processes in charm decays are much more highly suppressed by the GIM mechanism than the corresponding down-type quark decays because of the large top quark mass. Observation of $D^{+}$FCNC decays $D^{+} \rightarrow \pi^{+} l^{+} l^{-}$and $D^{+} \rightarrow K^{+} l^{+} l^{-}$could therefore provide indication of new physics or of unexpectedly large rates for long-distance SM processes like $D^{+} \rightarrow$ $\pi^{+} V, V \rightarrow l^{+} l^{-}$, with real or virtual vector meson $V$. Recently, CLEO-c report the branching fraction of the resonant decay $\mathcal{B R}\left(D^{+} \rightarrow\right.$ $\left.\pi^{+} \phi \rightarrow \pi^{+} e^{+} e^{-}\right)=(2.8 \pm 1.9 \pm 0.2) \times 10^{-6}$. The lepton-number-violating (LNV) or leptonflavor-violating (LFV) decays $D^{+} \rightarrow \pi^{-} l^{+} l^{+}$, $K^{-} l^{+} l^{+}$and $\pi^{+} \mu^{+} e^{-}$are forbidden in the SM. Past searches have set upper limits for the dielectron and dimuon decay modes [16]. In Table [5] and Table [6] the current limits and expected sensitivities at BES-III are summarized for $D^{+}$and $D^{0}$, respectively. Detailed description on rare charm decays can be found in references 35 36]. The charm meson radiative decays 
are also very important to understand final state interaction which may enhance the decay rates. In Ref. 3536 , the decay rates of $D \rightarrow V \gamma(V$ can be $\phi, \omega, \rho$ and $K^{*}$ ) had been estimated to be $10^{-5}-10^{-6}$, which can be reached at BES-III.

\section{Acknowledgment}

The author would like to thank David Asner for many useful discussions and comments.

\section{REFERENCES}

1. C. Aubin et al., Phys. Rev. Lett. 94 (2005) 011601; A. X. El-khadra et al. Phys. Rev. D64 (2001) 014502.

2. Internal Report, "The Preliminary Design Report of the BESIII Detector", IHEPBEPCII-SB-13.

3. R. Poling, Talk at the FPCP 2006 on "Hot Topics from CLEO-c" in Vancouver, April 9, 2006.

4. L. Gibbons, hep-ex/0107079

5. M. Gronau, Y. Grossman, and J. L. Rosner, Phys. Lett. B 508 (2001) 37.

6. D. M. Asner and W. M. Sun, Phys. Rev. D73 (2006) 034024.

7. P. Rubin et al., hep-ex/0604043

8. D. Scora and N. Isgur, Phys. Rev. D52 (1995) 2783; D. Melikhov and B. Stech, Phys. Rev. D62 (2000) 014006;A. Khodjamirian et al., Phys. Rev. D62 (2000) 114002.

9. H. G. Dosch et al., Phys. Rev. D65 (2002) 114002; M. Z. Yang, Phys. Rev. D73 (2006) 034027 .

10. V. V. Anisovich et al., Phys. Lett. B404 (1997) 166.

11. Th. Feldmann and P. Kroll, Phys. Rev. D58 (1998) 114006.

12. C. S. Kim and Y. D. Yang, Phys. Rev. D65 (2001) 017501.

13. P. Rubin, et al., Phys. Rev. Lett. 96 (2005)081802.

14. Q. He et al., Phys. Rev. Lett. 95 (2005) 121801.

15. M. B. Voloshin, Phys. Atom. Nucl. 68 (2005) 771.

16. S. Eidelman et al., Phys. Lett.B592 (2004) 1.
17. M. B. Voloshin, hep-ph/0602233

18. N. Brambilla et al., hep-ph/0412158 (2004); H. J. Lipkin, Phys. Lett. B179 (1986) 278.

19. T. M. Yan, Phys. Rev. D22 (1980) 1652; Y. P. Kuang and T. M. Yan, Phys. Rev. D24 (1981) 2874; Phys. Rev. D41 (1990) 155; Y. P. Kuang, Phys. Rev. D65 (2002) 094024. 20. J. L. Rosner, Phys. Rev. D64 (2001) 094002. 21. J. Z. Bai et al., Phys. Lett. B605 (2005) 63.

22. N. E. Adam et al., Phys. Rev. Lett. 96 (2006) 082004; G. S. Adam et al., Phys. Rev. D73 (2006) 012002; T. E. Coan et al., hep-ex/0509030 G. S. Huang et al., Phys. Rev. Lett. 96 (2006) 032003.

23. D. Besson et al., Phys. Rev. Lett. 96 (2006) 092002.

24. G. Rong, AIP Conf. Proc. 814 (2006) 414.

25. P. Wang et al., hep-ph/0512329

26. D. M. Asner, $D^{0}-D^{0}$ mixing minireview, in PDG2004 as in ref. [16.

27. F. Buccella et al., Phys. Lett. B379 (1996) 249.

28. B. Aubert et al., Phys. Rev. Lett. 95 (2005) 121802;K. Abe et al., hep-ex/0504013

29. A. Bondar et al., hep-ph/0510246

30. Letter of Intent for KEK Super-B Factory, KEK Report 2004-4.

31. Q. He et al., Phys. Rev. Lett. 95 (2005) 221802.

32. J. M. Link et al., Phys. Lett. B572 (2003) 21.

33. E. M. Aitala et al., Phys. Lett. B462 (1999) 401.

34. P. L. Frabetti et al., Phys. Lett.B398 (1997) 239.

35. G. Burdman et al., Phys. Rev. D52 (1995) 6383; Phys. Rev. D66 (2002) 014009.

36. G. Burdman and I.Shipsey, Ann. Rev. Nucl. Part. Sci. 53 (2003) 431; S. Bianco, F. L. Fabbri, D. Benson and I. Bigi, Riv. Nuovo Cim. 26N7 (2003) 1.

37. T. E. Coan et al., Phys. Rev. Lett. 90 (2003) 101801.

38. A. Freyberger et al., Phys. Rev. Lett. 76 (1996) 3065; erratum, Phys. Rev. Lett. 77 (1996) 2147.

39. A. Korn et al., hep-ex/0305054

40. K. Kodama et al., Phys. Lett. B345 (1995) 85. 